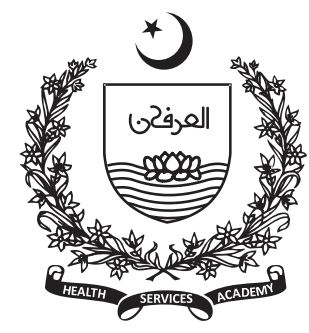

Aga Khan University

Corresponding Author: Sana Farrukh khan Email:

sana.iqbal2@scholar.aku.edu

\section{School Health Services (SHS): A Need in the Context of Pakistan Case Study}

\author{
Sana Farrukh Khan, Afshan Murad, Rozina Somani, Khairunnissa Hooda, \\ Salma Rattani
}

\section{Abstract}

School Health Services (SHS) are school health programs that promote health in school children. The value of SHS is recognized globally and practices around SHS vary with different regions. Its positive outcomes are already acknowledged by high-income countries, where education ministries are typically involved in cultivating comprehensive School Health Services. Lowincome countries remain hesitant to implement SHS, due to various constraints. In Pakistan, SHS have been introduced and restructured several times, but never comprehensively executed. Private schools in Pakistan offer better health programs than public schools but none of the schools follow standardized school health guidelines. This paper aims to analyze the role of SHS in Pakistan and provide several recommendations regarding the establishment of SHS in local schools. The proposed suggestions revolve around establishing model clinics in schools, which would be central to subsequent school-based health programs in Pakistan. If properly designed and equipped, school clinics can support a wide range of health outcomes, from emergency first aid care, to immunizations, to health education. The involvement of ministries and national authorities is encouraged for smooth delivery of SHS and compliance with public health guidelines.
A schoolboy was injured during his Physical Education (PE) class. He complained of pain in his right forearm and could not move his affected extremity. The PE teacher, who was responsible for first aid, asked him to rest his arm and go to class. Later, the child complained of severe pain, but no medication was given to him, due to the lack of School Health Services (SHS) including a school nurse. The boy remained in class for the rest of the day and could not rest because the school does not have a sick bay or school clinic. His mother was informed about his injury when she came to pick the child up from school. She took her son to a community clinic where an X-ray was recommended. The boy was diagnosed with a simple fracture of the radial ulna bone. However, the fracture required surgical treatment due to increased swelling, frequent mobility of the arm, and a delay in delivery of basic first aid care. Sadly, this is not a unique scenario in the context of Pakistan, where structured SHS are missing in many public and private schools. This paper will discuss the significance of SHS globally and specific to the context of Pakistan. It will also provide recommendations for establishing functional SHS in Pakistani schools.

\section{Introduction}

SHS is defined as a coordinated health program that promotes health in school children, allows for early detection and screening of health conditions, and ensures the continuum of care (Khalid, 2017). SHS is part of the public health sector, and this service requires a comprehensive program to cater the needs of the school community. The services included in SHS are health-promoting facilities such as emergency first aid, screening for diseases, health education, immunization, and well-being counseling for school children. Availability of these services promotes better health outcomes among school children. Indeed, a school is an ideal setting for promoting health because long-term behaviors and routines are often established during childhood. Moreover, students can acquire knowledge and experiences which they can then pass along to their family members. These services can also reduce the national disease burden due to increased awareness and behavior changes from an early age (Tiu et al., 2019). Beyond this, academic performance is directly related to good health. Healthy individuals are more likely to achieve academically, and SHS play 
a pivotal role in promoting good health among children (Auld et al., 2020). Students with special educational needs are also directly benefited by the presence of SHS in schools because they can be appropriately supported in their learning without exacerbating existing health problems. Similarly, students with chronic conditions such as asthma, allergies, and other disorders can receive assistance from school nurses if their symptoms become aggravated (Osian, Ehwarieme \& Igbinoba 2020). The World Health Organization (WHO) and other global organizations have highlighted the significance of SHS in the physical and educational wellbeing of students (Khalid, 2017). However, despite this international support and all of the positive outcomes to recommend SHS, most schools in Pakistan merely assign a teacher to provide onsite first aid and, ultimately, the School Health Services policies have not been implemented in many regions in Pakistan (Qureshi et al., 2018).

The SHS remains a neglected part of the primary health care sector of Pakistan. As a result, the provision of school clinics is uncommon in government run schools in Pakistan. Meanwhile, in the private school system, SHS is available. However, they usually don't follow any pre-determined school health guidelines from the Ministry of Health and some private schools operate health services organized by the school administration with limited external oversight (Uttanwalla \& Inayat 2019).

\section{Background}

Across the globe, the notion of school health programs has a long history, particularly in high-income countries. In these countries, the concept of school health services emerged gradually, with the United States leading the charge by implementing school health interventions across the country. In 1995, the WHO launched the Global School Health Initiative, intended to promote a healthy education environment. Many developing countries subsequently adopted comprehensive school health programs as recommended by the WHO sustainable development agenda (WHO, 2017). Most of the countries in the Middle East and North Africa (MENA) regions embraced school health guidelines outlined by their respective Ministries of Health. According to the United Arab Emirates (UAE), health regulation school guidelines (2018), for instance, a "school system must have a school clinic facility to provide onsite care to students, routine monitoring of the health status, and emergency care to ensure the provision of SHS in school" (p.42). Meanwhile, in Pakistan, the establishment of school clinics has theoretically been a part of the government health infrastructure since 1952, but, in reality, there is a huge gap in the actual implementation of School Health Services. In 2008, the school clinics' development program was revived by the Punjab Health Sector Reforms Program (PHSRP), and clinics were rebuilt in government schools in Punjab province. However, none of the other Pakistani provinces have school clinics (Uttanwalla \& Inayat 2019). Recently, a few private schools in cities in Pakistan have implemented SHS but still, the standardized clinics with adequate operations including basic health provision, counseling, immunization, and referral are missing.

\section{Significance of School Health Services (SHS)}

A school clinic serves the purpose of providing School Health Services to children. Daily first aid, chronic illness management, mental health care, counseling, dental health, ocular health, nutrition education, health education, and health promotion, with an emphasis on prevention and early intervention are prioritized. Osian, Ehwarieme and Igbinoba (2020), contend that the fundamental intentions of school clinics are prevention, early detection, and correction of disease among school-going children. Students with chronic health conditions can be provided with timely treatment of disease symptoms during school hours, which may prevent further advancement of the disease. Chidiebere et al., (2016) have identified that school children face significant environmental and behavioral risks such as injuries which arise during play or as a result of a physical altercation with another child. These risks are sometimes harmful to their physical and mental health. Therefore, each school needs a medical facility with skilled healthcare professionals and requisite medical equipment. Moreover, school children are vulnerable to several health conditions such as behavioral crises, and accidental/intentional injuries. These health risks are often interrelated and have a common underlying cause that can affect the child's ability to learn (Auld et al., 2020).

\section{Recommendations}

To promote health and prevent illnesses in schoolchildren, there is an urgent need to imbed school clinics in Pakistani schools. There are some important factors we need to keep in mind while proposing the accessibility of SHS through model clinics in schools. The following section of the paper will outline some essential requirements of SHS in the context of Pakistan. These recommendations are intended to spark discussion amongst Ministry of Health officials and school administrators.

Need Analysis

It is important to identify the number of students attending each school, as well as the existing services available in schools. Some schools presently only offer 
first aid treatments for emergencies. Ideally, schools must provide preventive services, health education, and even immunization at school (Ogbe, 2019). The other important component to ponder is the age ranges of children attending the school, which can help inform the necessary health measures and the upcoming health challenges according to the agerelated needs of the schoolchildren.

\section{Budget}

When budgeting for a school clinic, there are two major categories to consider: the initial investment cost for establishing a facility and the ongoing or monthly allocation necessary to maintain the clinic. The onetime investment includes approval costs, construction of a new facility, and purchase of the required equipment. The allocated budget to start a new clinic might be considered costly but the investment is preliminary, and the same cost will not be needed on a regular basis. As for the ongoing budget, most of the money required would be for salaried professionals, such as nurses and doctors, working in school clinics. Monthly purchases of medications and supplies, along with administrative assistance would also be covered under the ongoing budgeting (Jansen et al., 2019). In addition, the ongoing budget should be designed to mitigate outbreaks and seasonal communicable diseases such as chickenpox, conjunctivitis, GastroIntestinal (GI) outbreaks, etc.

Location and Environment

A school's clinic should ideally be built on the ground floor, to cater to any physically challenged students or students injured during school hours. The space allocated should be based on the size of the school population, with ventilation and lighting considered when choosing/designing the clinic. In addition, a school clinic should ideally be located in a quiet place. In particular, it should not be near a playground or music room, in order to avoid disruptive noises for the sick students or clinic staff. Within the clinic itself, there are a variety of things to consider. For instance, the clinic should have an adequate number of beds or chairs, depending on the size of the school, as well as privacy curtains between the beds. According to School Health guidelines published in UAE Sharjah (2020), "The facility needs to maintain suitable lighting and utilities, including cooling or heating devices, hand wash stations, sinks, drains, and an adequate number of toilets" (p. 73). The facility should also include an appropriate number of electrical outlets and communication systems like a telephone and internet access. Storage facilities for medical equipment such as medicine, bandages, oxygen cylinders, glucometers and other items should be located within the clinic. Ideally, storage facilities for non-medical equipment such as stationary, toiletries, bed linen, and cleaning supplies will also be available within the clinic. Beyond these physical features, school clinics must also have a plan for the disposal of medical waste, to limit the spread of infections within the school population. The management of medical waste is a significant concern, due to the potentially high risks to human health and the environment (Kumar, Somrongthong \& Ahmed, 2016). Therefore, biohazards bins are required, and regular collections of medical waste should be arranged.

\section{Healthcare Professionals}

School clinics are usually solo practice clinics, managed by a single full- or part-time nurse who can work independently. A study by Biag et al., (2015), notes greater school staff satisfaction with the presence of full-time school nurses in school. For medical checkups and referrals, part-time doctors should also be hired. The part-time doctors' visit on routine basis and the doctor should be on call in case of emergencies. Telehealth or e-health applications can also help nurses and students to connect with specialized physicians whenever required. An admin representative to notify parents where necessary should also be included in the health team. This role can be served by the school receptionist or any other designated staff who has access to school directories of parents' contact details.

\section{Interdepartmental Collaboration}

School clinics should liaise with other departments within the school, to ensure the children's health is prioritized across departments. For instance, school clinics should collaborate with the special needs' programs, to provide holistic care. The canteen is another important partnership. School clinics should work with the canteen to promote healthy eating in school and ensure that junk food is not allowed in the canteen. Nurses and doctors working in school clinics can also collaborate with school policymakers. For example, they can help inform responses to unusual health-related circumstances, such as the COVID-19 outbreak, by helping to design innovative standard operating procedures (SOPs) and ensuring that these policies are followed at the individual, and institutional levels. Physical Social and Health Education (PSHE) would also benefit from being linked to SHS. This partnership would help school administrations emphasize different aspects of health eating, healthy learning, and health education, which ought to be central to the curriculum (Gulzar et al., 2017).

\section{Intersectoral Collaboration}

Ideally, an integrated SHS is underpinned by intersectoral collaboration, which ensures its compliance with government policies and practices. Therefore, school administrations, health authorities, 
and policymakers should work in collaboration to establish SHS in the Pakistani school system (Ramukumba et al., 2019). This collaboration will enable a standardized delivery of health care in schools. Once SHS has launched in schools, continued monitoring is very important. Therefore, the government should have an inspection mechanism to monitor the functioning of school clinics. Unfortunately, at present, the government does not have a structured monitoring system for existing school clinics.

\section{Conclusion}

In Pakistan, 41.16 million children are enrolled in schools and represent more than one-fourth of the country's population. There is a critical need to introduce comprehensive SHS in schools in Pakistan (Khalid, 2017), with the anticipated benefits extending to school going children, their families and beyond. SHS offers a variety of important outcomes. For instance, it can help ensure that students with special needs or complex medical histories receive holistic care. SHS can also help boost inoculation levels in Pakistan and support students to greater academic achievements. The recommendations outlined in this paper serve to inform the considerations each school should recognize, when opening a clinic. While this is not a straight-forward process, it is clearly a worthy one. The Ministry of Health should prioritize SHS by arranging for sustainable support from UNESCO, UNICEF, and the WHO.

\section{References}

1. Auld, M. E., Allen, M. P., Hampton, C., Montes, J. H., Sherry, C., Mickalide, A. D., \& Parson, K. (2020). Health literacy and health education in schools: collaboration for action. NAM Perspectives. https://doi.org/10.31478/202007b

2. Biag, M., Srivastava, A., Landau, M., \& Rodriguez, E. (2015). Teachers' perceptions of full-and part-time nurses at school. The journal of school nursing, 31(3), 183195.

3. Chidiebere, O. D., Thomas, U. O., Joy, E., Stanley, O. K., Ikenna, N. K., Uchenna, E., \& Is, A. N. (2016). The status of school health services: a comparative study of primary schools in a developing country. Am J Public Health Res, 4(2), 42-6.

4. Dubai Health Authority. (2020). Standards for Clinics in Educational and Academic Settings. Retrieved from:
https://www.dha.gov.ae/en/HealthRegulation/Docu ments/Standards\%20for\%20Clinics \% 20in \%20Education al\%20and \%20Academic\%20Settings.pdf

5. Gulzar, S. A., Nayani, P., Husain, S. S., \& Khan, A. (2017). Development of the School Health Education Program from the perspective of Comprehensive School Health Model: An example at a higher secondary school of Karachi, Pakistan. Taiwan Gong Gong Wei Sheng Za Zhi, 36(6), 545.

6. Jansen, D., Vervoort, J. P., Visser, A., Reijneveld, S. A., Kocken, P., de Lijster, G., \& Michaud, P. A. (2019). School Health Services. In Issues and Opportunities in Primary Health Care for Children in Europe: The Final Summarized Results of the Models of Child Health Appraised (MOCHA) Project (pp. 219-236). Emerald Publishing Limited

7. Khalid, S. T. (2017). "School Health Services." Journal of Islamabad Medical E Dental College 6(1): 1-2.

8. Kumar, R., Somrongthong, R., \& Ahmed, J. (2016). Effect of medical waste management trainings on behavior change among doctors versus nurses and paramedical staff in Pakistan. Journal of Ayub Medical College Abbottabad, 28(3), 493-496.

9. Osian, E. A., Ehwarieme, T. A., \& Igbinoba, O. (2020). Knowledge, Attitude and Utilization of School Health Services among Senior Secondary School Students in Egor Local Government Area, Benin City, Edo State. International Journal of tropical disease $\mathcal{E}$ health, 1-9. DOI: $10.9734 /$ ijtdh/2020/v41i1230336

10. Qureshi, F. M., Khalid, N., Nigah-e-Mumtaz, S., Assad, T., \& Noreen, K. (2018). First aid facilities in the school settings: Are schools able to manage adequately? Pakistan journal of medical sciences, 34(2), 272

11. Ramukumba, Tendani S., Richard M. Rasesemola, and Gert P. Matshoge. Compliance to the integrated school health policy: Intersectoral and multisectoral collaboration. Curationis 42, (1) 2019 1-8.

12. Uttanwalla, K. S. and S. Inayat. (2019). "Significance of School Health Nurse in Screening School Children in Islamabad Pakistan. Saudi Journal of Nursing and Health Care, 2(5) 168-171 " DOI:10.21276/sjnhc.2019.2.5.1

13. United Arab Emirates Ministry of Health \& Prevention. (2016). School clinic regulation ministry of health. Retrieved from: https://www.mohap.gov.ae/School\%20clinic \%20R egulation.pdf

14. World Health Organization. (2017). Global school health initiatives: achieving health and education outcomes: Report of a meeting, Bangkok, Thailand, 23-25 November 2015 (No. WHO/NMH/PND/17.7). World Health Organization. Retrieved from: http://apps.who.int/iris/handle/10665/259813 